\title{
Impact of delayed neutron constants on reactivity effects measured in a fast reactor
}

\author{
Antonín Krása ${ }^{1, *}$, Anatoly Kochetkov ${ }^{1}$, Nadia Messaoudi ${ }^{1}$, Alexey Stankovskiy ${ }^{1}$, Guido \\ Vittiglio $^{1}$, Jan Wagemans ${ }^{1}$ \\ ${ }^{1}$ Belgian Nuclear Research Centre SCK•CEN, Boeretang 200, 2400 Mol, Belgium
}

\begin{abstract}
Delayed neutron parameters of fast VENUS-F reactor core configurations are determined with Monte Carlo calculations using various nuclear data libraries. Differences in the calculated effective delayed neutron fraction and the impact of the delayed neutron data (6- or 8-group precursors) that are applied in the experimental data analysis on the measured reactivity effects are studied. Considerable differences are found due to application of ${ }^{235} \mathrm{U}$ and ${ }^{238} \mathrm{U}$ delayed neutron data from JEFF, JENDL and ENDF evaluations.
\end{abstract}

\section{Introduction}

Analysis of experimental data measured in numerous nuclear reactor physics experiments require the knowledge of core averaged kinetic parameters. Deterministic [1] or Monte Carlo methods [2,3] can be used to calculate the effective delayed neutron

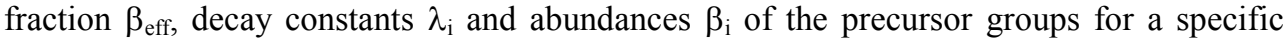
reactor core configuration as they depend on fuel composition and neutron spectrum.

Evaluated delayed neutron data (delayed neutron multiplicity $v_{\mathrm{d}}$, half-lives and abundances of precursors, delayed neutron spectra $\chi_{\mathrm{d}}$ ) are available in general purpose nuclear data libraries (e.g. JEFF, JENDL, ENDF). The delayed neutron spectra are given in energy-group format (usually 16 groups of Hansen-Roach set [4]). The evaluated decay constants and abundances are given in time-group format (usually 8 groups [5] or 6 groups [6]). The group constants (as well as the fission yields) are evaluated at three energies: thermal, fast (fission), high (fusion). This study is focused on fast fission in ${ }^{235} U$ and ${ }^{238} U$.

The influence of delayed neutron data on thermal reactor parameters has been widely investigated (e.g. [7,8]). In fast reactors the influence of delayed neutron spectra was studied $[9,10]$, benchmarks for $\beta_{\text {eff }}$ were carried out at MASURCA and FCA critical facilities [11] and used for adjustment of delayed neutron yields [12]. In this paper, we describe the impact of delayed neutron data on the analysis of reactivity measurements at the fast zero-power VENUS-F reactor located at the Belgian Nuclear Research Center $\mathrm{SCK} \cdot \mathrm{CEN}$. VENUS-F has been extensively used for fast neutron data validation [13-17] serving as a mockup of the MYRRHA fast reactor/accelerator driven system $[18,19]$. Sensitivity and uncertainty analysis of $\beta_{\text {eff }}$ were recently studied for the specific cases of the VENUS-F [20] and MYRRHA reactors [21].

\footnotetext{
* Corresponding author: antonin.krasa@sckcen.be
} 


\section{Delayed neutron data}

The half-lives and abundances of delayed neutron precursors are represented either with 6 groups (isotope-dependent half-lives) or 8 groups (identical half-lives for all the fissionable isotopes). Each nuclear data library makes its own best estimate based on various evaluated data available for each of the fissile and fertile isotopes in three energy regions. The current status of the delayed neutron parameters of ${ }^{235} \mathrm{U}$ and ${ }^{238} \mathrm{U}$ (relevant for the present study) adopted in the main nuclear data libraries is the following.

The JEFF library (since version 3.1) [22] uses Spriggs data (both decay constants and abundances) in 8-group format [4,5]. The JENDL library (since version 3.2) [23] uses the 6-groups data: decay constants of Keepin [6] and abundances evaluated on the basis of a large set of experimental and evaluated data. The ENDF library (since version VII.0) [24] uses the 6-groups data that come from summation calculations, which use decay constants and delayed neutron emission probabilities from the evaluated experimental data file NuBase2003 [25]; if not available, the data are calculated using quasi-particle randomphase approximation and the statistical gross theory [26]. One of the most remarkable features in ENDF is the remarkably short first group half-life compared to the other two evaluations, see Table 1. Other libraries like BROND-3.1, CENDL-3.1, TENDL-2017 adopted the same decay constants as in ENDF.

There are no uncertainties of the decay constants and group abundances in the evaluated data libraries. Only the original Spriggs data [5] contain uncertainties of the group abundances (group half-lives are fixed, thus, their uncertainties are reflected in the uncertainties of the abundances), which further leads to the uncertainty of the average halflife of the delayed neutrons $\left(9.10 \mathrm{~s} \pm 1 \%\right.$ for fast ${ }^{235} \mathrm{U}$ and $5.3 \mathrm{~s} \pm 3 \%$ for fast ${ }^{238} \mathrm{U}$ fissions).

Table 1. Half-lives of precursor groups in JEFF-3.1.2, JENDL-4.0, ENDF/B-VII.1.

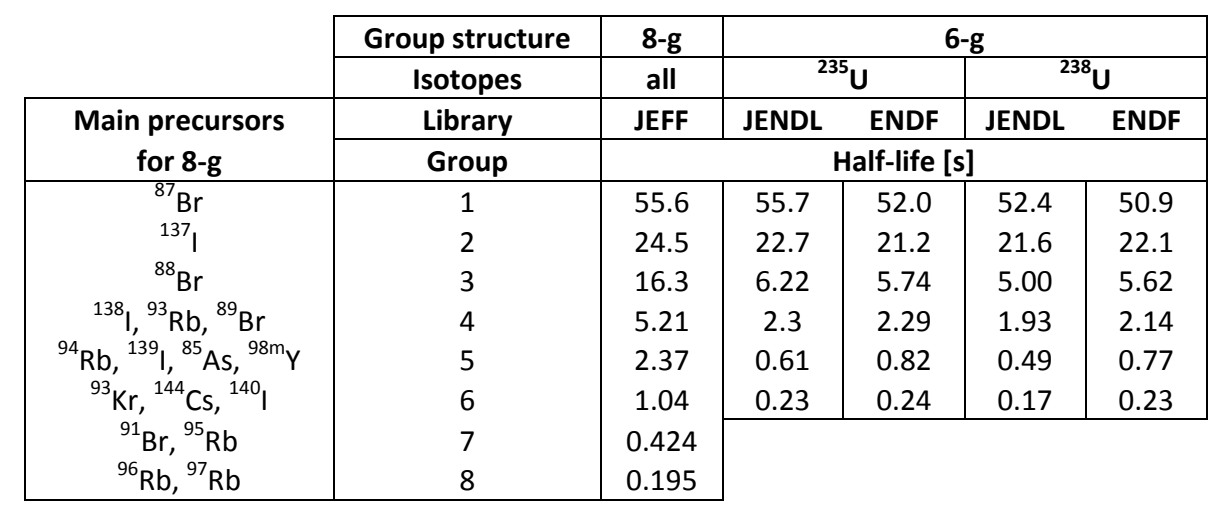

Although the group constants evaluated for thermal (25 meV) and high (14 MeV) energy have unambiguously defined energy, the mean energy of the fast spectrum specified in the evaluations is not always clear because of a wide variation in the mean energies of the experimental setups used in integral data studies. The ${ }^{235} \mathrm{U}$ evaluations in the fast spectrum correspond to a mean energy of hundreds of $\mathrm{keV}$ (some evaluations refer to 200 $\mathrm{keV}$ [4], $400 \mathrm{keV}$ [22], $500 \mathrm{keV}$ [24] or a range from $50 \mathrm{keV}$ to $7 \mathrm{MeV}$ [23]). However, the fast spectrum values for ${ }^{238} \mathrm{U}$ rather correspond to energies slightly above $1 \mathrm{MeV}$ (although the evaluations again refer to 400 or $500 \mathrm{keV}$, which is not realistic due to the ${ }^{238} \mathrm{U}$ fission threshold energy). The energy dependence of the group decay constants was extensively measured [27]. 
In general, the delayed neutron production has a non-trivial energy dependence, especially above $1 \mathrm{MeV}$, see ${ }^{235} \mathrm{U}$ in Fig. 1 . Below $10 \mathrm{keV}, v_{\mathrm{d}}$ is constant and JENDL and ENDF give nubar about $2 \%$ smaller than JEFF. Between 10 and $50 \mathrm{keV}, v_{\mathrm{d}}$ increases according to JENDL and ENDF, while it stays constant in JEFF evaluation. Between 50 $\mathrm{keV}$ and $1 \mathrm{MeV}$, ENDF gives $v_{\mathrm{d}}$ about 2-3\% bigger than JEFF and JENDL. The sharp decrease of $v_{\mathrm{d}}$ above $4 \mathrm{MeV}$ is likely to be caused by the second chance fission process. The $v_{\mathrm{d}}$ covariances in JENDL lead to $3.5 \%$ uncertainty below $20 \mathrm{keV}$ and $7 \%$ uncertainty between $20 \mathrm{keV}-3 \mathrm{MeV}$, which is significantly bigger than the differences between various evaluations.

The delayed neutron production in ${ }^{238} \mathrm{U}$ is about 3 times bigger than in ${ }^{235} \mathrm{U}$ with differences up to $5 \%$ between the evaluations.

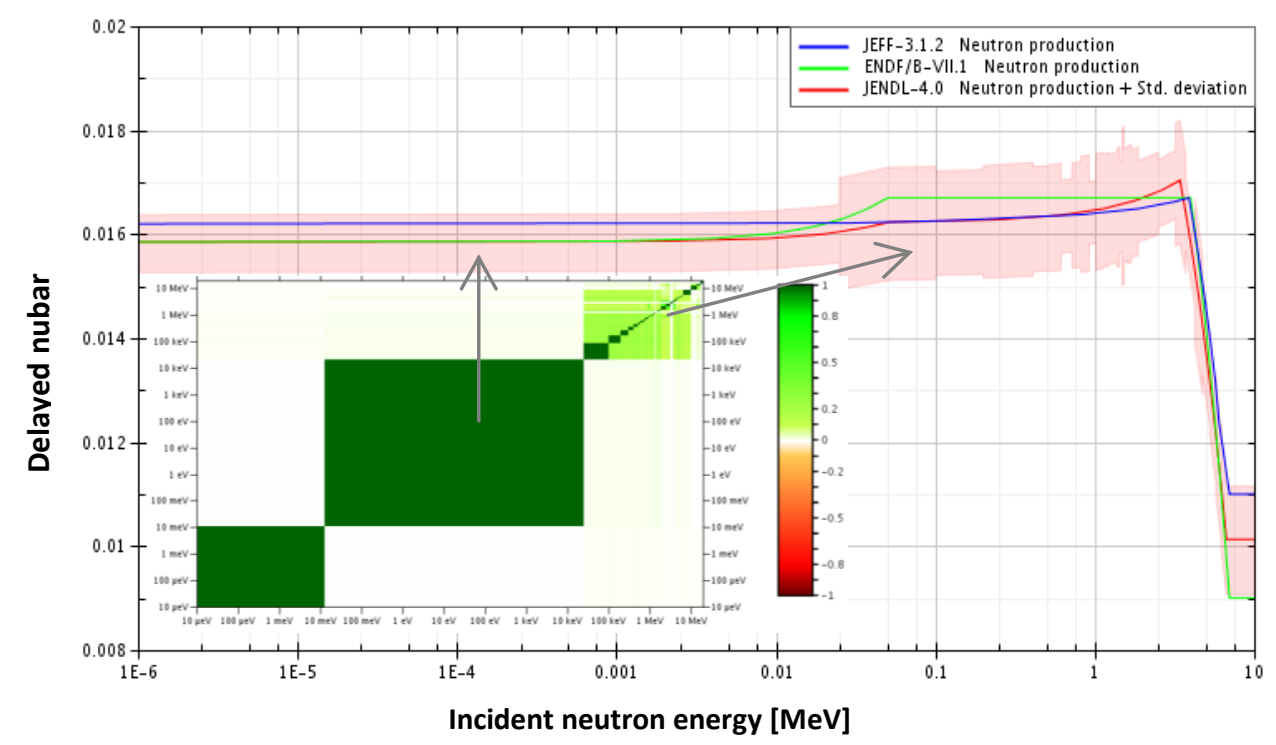

Fig. 1. The evaluated average number of delayed neutrons for ${ }^{235} \mathrm{U}(\mathrm{n}, \mathrm{f})$. The inset shows covariances from JENDL-4.0 and the arrows show where they contribute to the uncertainties.

\section{Experiment}

VENUS-F [28] is a fast zero-power reactor loaded with $30 \%$ wt. metallic uranium fuel. The core configuration presented in this paper used solid $\mathrm{Bi}$ as a coolant simulator and lead as reflector, see Fig. 2 (detailed description of fuel assembly composition and the entire core can be found in [15]).

For measurements of reactivity effects, either the compensation method [29] or the MSM method [30] are used. Both require the application of the delayed neutron parameters. The former through the calibration of control rods with a period measurement (the inhour equation). The latter requires the determination of a reference sub-critical state. For that purpose, a rod drop method is applied (see further).

The VENUS-F reactor was operated in a critical state at a stable power for sufficiently long time for the neutron population and precursor concentrations to reach equilibrium (about $20 \mathrm{~min}$ ). Fission chambers [31] inserted in experimental channels in the reflector (see Fig. 2 left) were used to record a constant count rate $n_{0}$.

Then, an absorber rod (made of $\mathrm{B}_{4} \mathrm{C}$ powder) located in the reflector (see Fig. 2) was rapidly inserted (the rod drop took $0.4 \mathrm{~s}$ ). Detector count rates $n(t)$ were measured as a 
function of time after the rod drop $(t=0)$ until counting only background counts, i.e. the complete decay of the precursors, see Fig. 3 left.

The reactivity of the sub-critical configuration was determined using the integral counting method:

$$
-\frac{\rho}{\beta_{\mathrm{eff}}}=\frac{n_{0} \sum_{i=1}^{G} \frac{\alpha_{i}}{\lambda_{i}}}{\int_{0}^{\infty} n(t) d t}
$$

that requires the knowledge of the effective delayed neutron fraction $\beta_{\text {eff }}$ and the relative abundances of each precursor group $\alpha_{i}=\beta_{\text {eff }}^{i} / \beta_{\text {eff }}$ (the number of groups being $G=6$ or 8 ) characterized by the decay constants $\lambda_{i}$ (calculated using the Monte Carlo method, see next section).

The expression $\sum \alpha_{i} / \lambda_{i}$ in equation (1) represents the mean life time of a delayed neutron $\tau_{\mathrm{d}}=1 / \lambda_{\mathrm{d}}=T_{1 / 2} / \ln (2)$. Its uncertainty combined with the uncertainty of the counting statistics of $n_{0}$ and the integral of $n(t)$ after the rod drop contribute to the total uncertainty of the measured reactivity in units of $\$$. The uncertainty of $\beta_{\text {eff }}$ needs to be added if the reactivity in units of pcm is required.
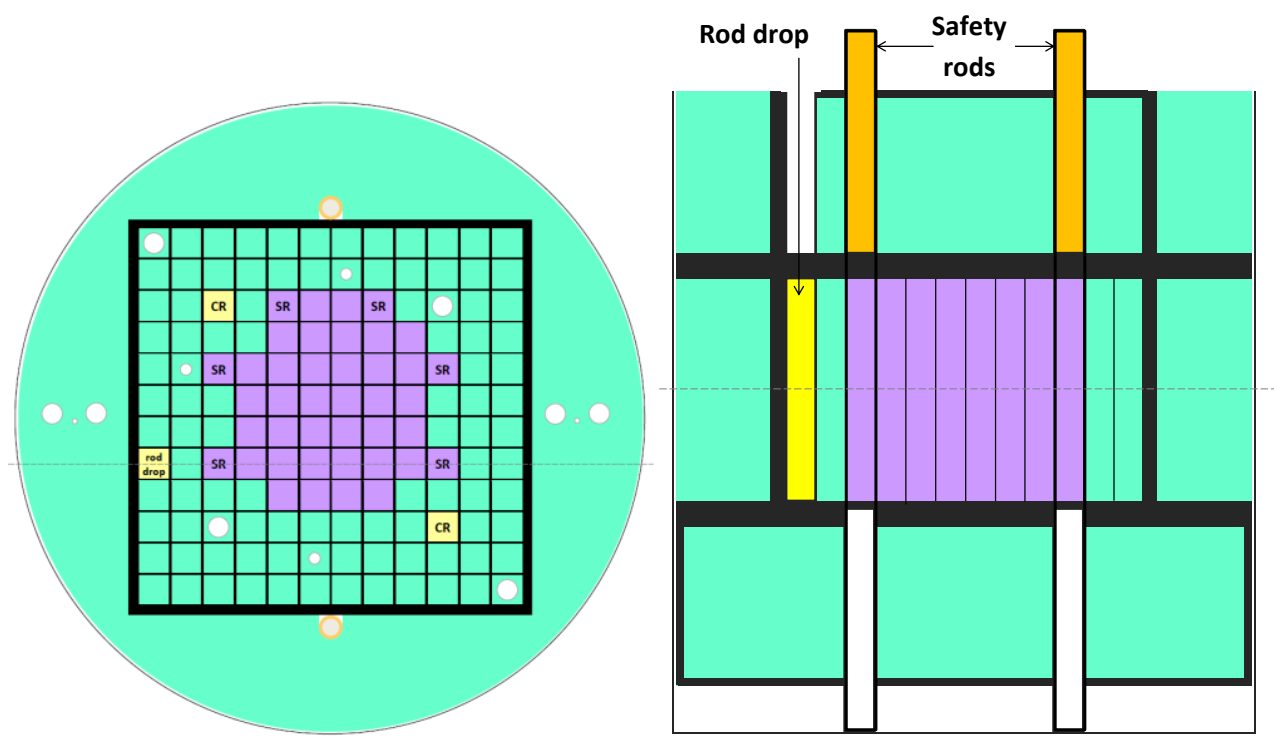

Fig. 2. Left: radial cut through the VENUS-F reactor configuration discussed in this paper. The cut passes through the core midplane (marked with a dashed line in the right-hand side figure). The assembly pitch is $8 \mathrm{~cm}$, both the core diameter and height are $160 \mathrm{~cm}$.

Right: axial cut through the core at the plane passing the rod drop and two safety rods (marked with a dashed line in the left-hand side figure). The depicted situation is after the rod was dropped (i.e. it is fully inserted in the core). Fuel assemblies - violet, $\mathrm{B}_{4} \mathrm{C}$ control rods (CR) and rod drop system yellow, $\mathrm{B}_{4} \mathrm{C}$ safety rods (SR) - orange, lead reflector - green, experimental channels with fission chambers - white circles, stainless steel casing - black. 

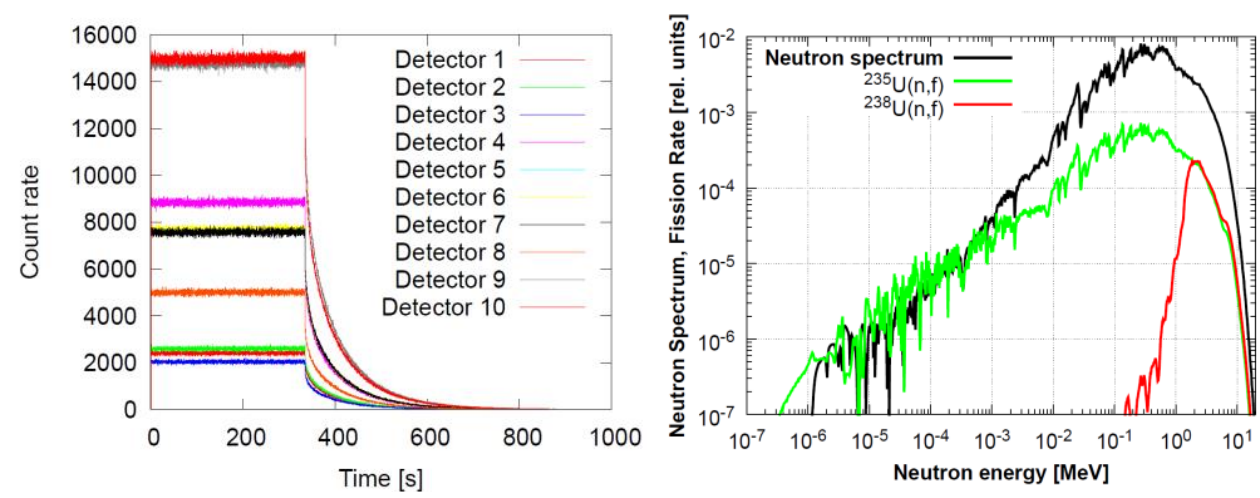

Fig. 3. Left: Detector count rates during a stable critical operation, rod drop and decay.

Right: MCNP simulation of neutron spectrum and energy dependence of the fission rates in fuel.

\section{Calculation tools}

The MCNP5-1.60 code [32] was used to calculate the kinetic parameters averaged over the VENUS-F core (i.e. spectrum and isotope averaged) with the iterated fission probability (IFP) approach [33]. The KOPTS card [34] with the number of cycles in blocks for adjoint weighting equal to 10 was used. Each criticality calculation consisted of 1850 active cycles preceded by 50 inactive, each of them having $10^{6}$ neutrons. Such high number of histories was chosen to suppress the statistical uncertainties of the calculated kinetic parameters. The uncertainty of the calculated $\beta_{\text {eff }}$ is 2 pcm (i.e. $0.3 \%$ ) in all cases.

It was tested at various VENUS-F core configurations that the IFP approach gives the same result of $\beta_{\text {eff }}$ as the prompt method [2]. An extensive verification of the Serpent-2 code against MCNP performed with the first VENUS-F critical core [35] provided a confidence in consistency of results, among others, $\beta_{\text {eff }}$ results of both codes agree within $1 \sigma$.

Cross-sections from the following evaluated nuclear data libraries were used in the MCNP calculations:

- JEFF-3.1.2 (released in 2012 [36]) that is taken as a reference library in the next section; the same delayed neutron data were adopted in the newest release JEFF-3.3 (Nov 2017 [37]);

- JENDL-4.0 (released in 2010 [23]);

- ENDF/B-VII.1 (released in 2011 [24]) - the same delayed neutron data were adopted in the newest release ENDF/B-VIII.0 (Feb 2018 [38]), the only difference being the added $v_{\mathrm{d}}$ covariances.

\section{Results}

First, the core averaged kinetic parameters calculated with different libraries are compared. Then their influence on the measured reactivity effects is discussed and the experimental results are compared with the MCNP calculations of the reactivity effects.

\subsection{Effective delayed neutron fraction}

The calculation with JEFF-3.1.2 gives $\beta_{\text {eff }}=(741 \pm 2)$ pcm, while JENDL-4.0 and ENDF/B-VII.1 give 1\% (i.e. 3-4 $\sigma$ ) smaller values. In one of the previous VENUS-F cores 
the kinetic parameters were measured [39]. All the calculations agree with the experimental value $\beta_{\text {eff }}=(730 \pm 11) \mathrm{pcm}$ within the uncertainties.

That is the situation if cross sections and delayed neutron parameters of all the isotopes are used from a single library. In order to distinguish between the impact of both uranium isotopes and to investigate whether the cross sections have any impact on the core averaged kinetic parameters, new calculations were performed having JEFF-3.1.2 as a reference library and altering either the cross sections and the delayed neutron data or only the delayed neutron data of JEFF-3.1.2 with those of ENDF/B-VII.1 and JENDL-4.0 for ${ }^{235} \mathrm{U}$ and ${ }^{238} \mathrm{U}$ isotopes separately, see Table 2 .

Replacing any of the JEFF-3.1.2 ${ }^{235} \mathrm{U}$ or ${ }^{238} \mathrm{U}$ cross-sections or the delayed neutron data with those of JENDL-4.0 (while keeping everything else from JEFF-3.1.2) leads to the $\beta_{\text {eff }}$ value decreases by $1 \%$. No statistically significant influence of the ${ }^{235} \mathrm{U}$ and ${ }^{238} \mathrm{U}$ cross sections on $\beta_{\text {eff }}$ was observed.

If the delayed neutron data from ENDF/B-VII.1 are used for ${ }^{235} \mathrm{U}$ or ${ }^{238} \mathrm{U}$, then the $\beta_{\text {eff }}$ value increases by $1 \%$ or decreases by $2 \%$, respectively. Such a strong influence of ${ }^{238} \mathrm{U}$ can be explained by the fast neutron spectrum (about $10 \%$ of fissions in the fuel happens in ${ }^{238} \mathrm{U}$, see Fig. 3 right) and the bigger delayed neutron multiplicity than ${ }^{235} \mathrm{U}$. The opposite effects of ${ }^{235} \mathrm{U}$ and ${ }^{238} \mathrm{U}$ partially cancel out and lead to a $\beta_{\text {eff }}$ value that is $1 \%$ smaller when ENDF/B-VII.1 is used for all the isotopes compared to JEFF-3.1.2.

Table 2. Effective delayed neutron fraction calculated with MCNP using JEFF-3.1.2, JENDL-4.0, ENDF/B-VII.1 libraries for cross sections and delayed neutron parameters.

\begin{tabular}{|c|c|c|c|c|c|c|c|c|}
\hline \multirow{3}{*}{$\frac{{ }^{235} U}{J E F F}$} & \multicolumn{2}{|c|}{ Cross-sections } & \multicolumn{2}{|c|}{ DN data } & \multirow{2}{*}{\multicolumn{3}{|c|}{$\begin{array}{c}\beta_{\text {eff }} \\
{[\mathrm{pcm}]}\end{array}$}} & \multirow{2}{*}{$\begin{array}{l}\text { Diff from } \\
\text { reference }\end{array}$} \\
\hline & ${ }^{238} \mathrm{U}$ & all other isotopes & ${ }^{235} \mathrm{U}$ & ${ }^{238} \mathrm{U}$ & & & & \\
\hline & JEFF & JEFF & JEFF & JEFF & 741 & \pm & 2 & $0.0 \%$ \\
\hline ENDF & ENDF & ENDF & ENDF & ENDF & 733 & \pm & 2 & $-1.1 \%$ \\
\hline JENDL & JENDL & JENDL & JENDL & JENDL & 734 & \pm & 2 & $-0.9 \%$ \\
\hline ENDF & JEFF & JEFF & ENDF & JEFF & 747 & \pm & 2 & $0.8 \%$ \\
\hline JEFF & JEFF & JEFF & ENDF & JEFF & 748 & \pm & 2 & $0.9 \%$ \\
\hline JEFF & ENDF & JEFF & JEFF & ENDF & 726 & \pm & 2 & $-2.0 \%$ \\
\hline JEFF & JEFF & JEFF & JEFF & ENDF & 727 & \pm & 2 & $-1.9 \%$ \\
\hline JENDL & JEFF & JEFF & JENDL & JEFF & 734 & \pm & 2 & $-0.9 \%$ \\
\hline JEFF & JEFF & JEFF & JENDL & JEFF & 731 & \pm & 2 & $-1.3 \%$ \\
\hline JEFF & JENDL & JEFF & JEFF & JENDL & 735 & \pm & 2 & $-0.8 \%$ \\
\hline JEFF & JEFF & JEFF & JEFF & JENDL & 733 & \pm & 2 & $-1.1 \%$ \\
\hline
\end{tabular}

\subsection{Precursor groups}

Core averaged abundances of delayed neutron precursors calculated using the three main libraries are compared in Fig. 4. Only the $1^{\text {st }}$ and the $4^{\text {th }}$ group from ENDF and JENDL have similar values. The $2^{\text {nd }}$ and the $3^{\text {rd }}$ group in JENDL have abundances bigger by $20 \%$ than in ENDF, while the $5^{\text {th }}$ and the $6^{\text {th }}$ group in ENDF have abundances bigger than JENDL by $20 \%$ and $40 \%$, respectively. ENDF has thus more weight on the shorterliving precursors and, as a consequence, the average delayed neutron half-life is shorter: $7.05 \mathrm{~s}$ for ENDF, $8.22 \mathrm{~s}$ for JENDL, $8.18 \mathrm{~s}$ for JEFF.

Mixing the delayed neutron data for fissionable isotopes in 6 and 8 groups in one calculation, as it was done in the previous section, can lead to absurd core averaged group half-lives. JEFF-3.1.2 used as a reference has the same half-lives of the 8 groups for both ${ }^{235} \mathrm{U}$ and ${ }^{238} \mathrm{U}$. If ENDF or JENDL data in 6 groups are applied for one of the isotopes, then 
the core averaged half-lives of the $1^{\text {st }}-6^{\text {th }}$ groups change, while those of the $7^{\text {th }}$ and the $8^{\text {th }}$ groups remain the same. That can cause the $6^{\text {th }}$ group having shorter half-life than the $7^{\text {th }}$ group, see example in Table 3.

In fact, such mixing is not an artificial thing. In JEFF, delayed neutron data in the 8 groups format are used for the well-known fissionable isotopes, however, for some other isotopes the delayed neutron data are evaluated in the 6 groups format [37]. Even in one of the test versions of JEFF-3.3, the 6 groups format was considered for ${ }^{235} \mathrm{U}$ and the 8 groups format for ${ }^{238} \mathrm{U}$ [37].

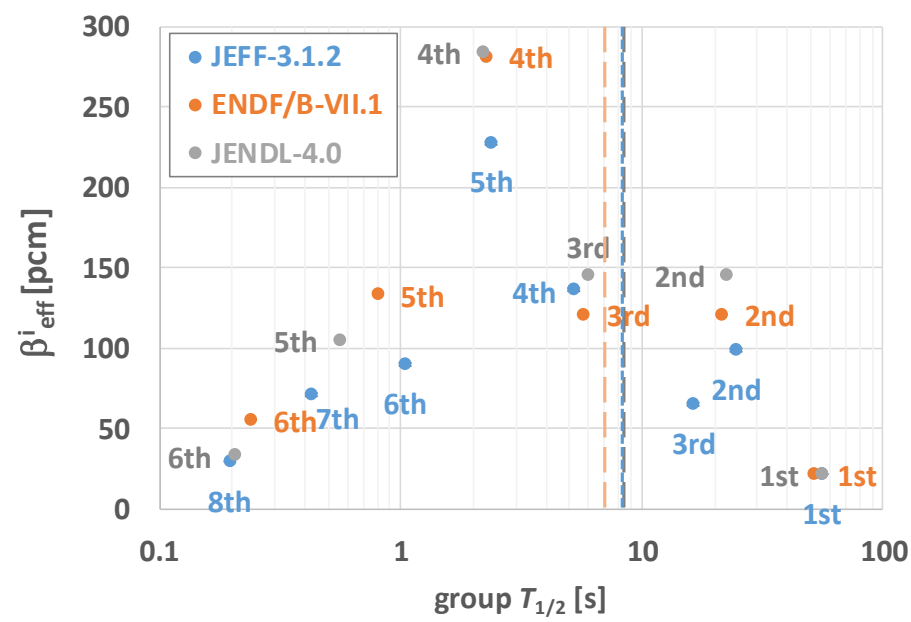

Fig. 4. Core averaged abundances of delayed neutron precursors in dependence of the half-life (in log-scale). Calculated with MCNP using JEFF-3.1.2 (8 groups), JENDL-4.0 and ENDF/B-VII.1 (6 groups) libraries. The uncertainties are $\leq 1 \mathrm{pcm}$ in all cases. The average half-life of each of the three data sets is shown with a dashed line.

Table 3. Core averaged group half-lives calculated with MCNP using 8 groups format for both fissionable isotopes (first row) or combining 8 groups for ${ }^{238} \mathrm{U}$ and 6 groups for ${ }^{235} \mathrm{U}$ (second row). For the latter, the $6^{\text {th }}$ group has shorter half-life than the $7^{\text {th }}$ group.

\begin{tabular}{|cc|ccccccccc|}
\hline & \multicolumn{8}{|c|}{ DN group } \\
\hline \multicolumn{2}{|c|}{ DN data library } & 1st & 2nd & 3rd & \multicolumn{1}{c|}{$4^{\text {th }}$} & 5th & 6th & 7th & 8th \\
\hline $235 \mathrm{U}$ & $238 \mathrm{U}$ & \multicolumn{1}{|c}{ Half-life [s] } \\
\hline JEFF (8-g) & JEFF (8-g) & 55.6 & 24.5 & 16.3 & 5.21 & 2.37 & 1.04 & 0.424 & 0.195 \\
ENDF (6-g) & JEFF (8-g) & 52.2 & 21.6 & 5.96 & 2.41 & 1.05 & 0.374 & 0.424 & 0.195 \\
\hline
\end{tabular}

\subsection{Reactivity effects}

The evaluated delayed neutron data have impact on the measured rod drop reactivity, see equation (1). The reactivity (in $\$$ ) is linearly proportional to the mean delayed neutron life time $\tau_{\mathrm{d}}$. As shown in the previous section, $\tau_{\mathrm{d}}$ of the investigated VENUS-F core has almost the same values (difference of $0.6 \%$ ) when calculated with 8 groups of JEFF and 6 groups of JENDL delayed neutron precursor data. Therefore, the application of JEFF and JENDL lead to the same measured reactivity in $\$$ and ENDF gives about $14 \%$ smaller reactivity in $\$$. For reactivity in pcm, the difference between JEFF and the other two libraries increases by $1 \%$, see section 5.1 . 
MCNP calculations of the rod drop are compared with the experimental results in Table 4. A small variation (not more than $2 \%$ ) in the calculated rod drop reactivity was found when different libraries were used in the calculations.

When delayed neutron data from JEFF or JENDL were applied in the analysis of experimental data, then the calculations underestimate such experimental results (by 6-8 $\%)$. In case of ENDF, the calculations overestimate the experiment by $6 \%$.

The core averaged delayed neutron data from mixed libraries (discussed in sections 5.1 and 5.2) were also applied to the experimental reactivity determination, see Table 4. Replacing the JEFF delayed neutron data of ${ }^{235} \mathrm{U}$ by JENDL does not change the reactivity value, while replacing by ENDF leads to a significant change and overestimation of the experiment by $9 \%$. On the contrary, replacing the JEFF delayed neutron data of ${ }^{238} U$ by either JENDL or ENDF leads to a perfect agreement between experiment and calculation.

Table 4. Comparison of the experimental and calculated rod worth when JEFF-3.1.2, JENDL-4.0 and ENDF/B-VII.1 libraries are utilized for experimental data analysis (eq. (1)) as well as for the MCNP calculations.

\begin{tabular}{|c|c|c|c|c|c|c|c|c|c|c|c|}
\hline XS library & \multicolumn{2}{|c|}{ DN data library } & \multicolumn{9}{|c|}{ Rod worth [pcm] } \\
\hline & $235 \mathrm{U}$ & $238 \mathrm{U}$ & \multicolumn{3}{|c|}{ EXP } & \multicolumn{3}{|c|}{ MCNP } & \multicolumn{3}{|c|}{$\mathrm{C} / \mathrm{E}$} \\
\hline JEFF & \multicolumn{2}{|c|}{ JEFF } & 194 & \pm & 4 & 178 & \pm & 2 & 0.92 & \pm & 0.02 \\
\hline ENDF & \multicolumn{2}{|c|}{ ENDF } & 164 & \pm & 3 & 174 & \pm & 2 & 1.06 & \pm & 0.02 \\
\hline JEFF & ENDF & JEFF & 165 & \pm & 3 & 179 & \pm & 2 & 1.09 & \pm & 0.02 \\
\hline JEFF & JEFF & ENDF & 175 & \pm & 4 & 174 & \pm & 2 & 0.99 & \pm & 0.02 \\
\hline JENDL & \multicolumn{2}{|c|}{ JENDL } & 192 & \pm & 4 & 181 & \pm & 2 & 0.94 & \pm & 0.02 \\
\hline JEFF & JENDL & JEFF & 188 & \pm & 4 & 179 & \pm & 2 & 0.95 & \pm & 0.02 \\
\hline JEFF & JEFF & JENDL & 176 & \pm & 4 & 177 & \pm & 2 & 1.00 & \pm & 0.02 \\
\hline
\end{tabular}

\section{Conclusion}

The rod drop experiment performed at the fast VENUS-F core was analysed using core averaged delayed neutron parameters calculated with MCNP using the JEFF-3.1.2 (8-group precursors), JENDL-4.0 and ENDF/B-VII.1 (6-group precursors) libraries. Considerably discrepant experimental results (up to $15 \%$ difference) were observed.

Application of the JEFF-3.1.2 or JENDL-4.0 delayed neutron data leads to $\mathrm{C} / \mathrm{E}<1$, while ENDF/B-VII.1 leads to $\mathrm{C} / \mathrm{E}>1$. Similar trends (up to 10-15\%) were observed in other VENUS-F cores (the same fuel, slightly modified neutron spectrum).

Although the best agreement of experiments and calculations was reached with ${ }^{235} \mathrm{U}$ delayed neutron data from JEFF-3.1.2 combined with ${ }^{238} \mathrm{U}$ delayed neutron data from either JENDL-4.0 or ENDF/B-VII.1, mixing 6-group and 8-group time structure can lead to unphysical distribution of decay constants among the precursor groups.

As a next step, the impact of delayed neutron data on the reactivity effects in the MYRRHA core with MOX fuel will be investigated.

The first author acknowledges D. Foligno for fruitful discussion on the topic of delayed neutron measurements and evaluations.

\section{References}

1. M. Carta et al., Sci. Technol. Nucl. Ins., Article ID 584256 (2011) 
2. R. K. Meulekamp, S. C. van der Marck, Nucl. Sci. Eng. 152, 142-148 (2006)

3. B. Kiedrowski, F. Brown, P. Wilson, LA-UR-09-06786 (2009)

4. G. Rudstam et al., NEA/WPEC-6, Int. Evaluation Cooperation, Vol. 6 (2002)

5. G. D. Spriggs, J.M. Campbell, V. M. Piksaikin, Prog. Nucl. Energy 41, 223-251 (2002)

6. G. R. Keepin, T. F. Wimett, R. K. Zeigler, Phys. Rev. 107, 1044 (1957)

7. B. Geslot, C. Jammes, B. Gall, Ann. Nucl. Energy 34, 652-660 (2007)

8. A. Zoia et al., Ann. Nucl. Energy 110, 11-24 (2017)

9. E. Kiefhaber, Nucl. Sci. Eng. 111, 197-204 (1992)

10. S. Das, Nucl. Sci. Eng. 122, 344-358 (1996)

11. S. Okajima et al., Prog. Nucl. Energy 41, 285-301 (2002)

12. T. Sakurai, S. Okajima, J. Nucl. Sci. Technol. 39, 19-30 (2002)

13. A. Kochetkov et al., EPJ Web of Conferences 146, 06007 (2017)

14. A. Krása et al., EPJ Web of Conferences 146, 06019 (2017)

15. A. Krása et al., ASTM STP1608, 286-295 (2018)

16. E. Fridman, A. Kochetkov, A. Krása, Ann. Nucl. Energy 108, 239-252 (2017)

17. M. Sarotto et al., Ann. Nucl. Energy 121, 626-637 (2018)

18. G. Van den Eynde et al., Proc. TCADS-3, 141-150 (2016)

19. A. Stankovskiy et al., Nucl. Data Sheets 118, 513-515 (2014)

20. H. Iwamoto, A. Stankovskiy, L. Fiorito, G. Van den Eynde, J. Nucl. Sci. Technol. 55, 539-547 (2018)

21. I. A. Kodeli, Ann. Nucl. Energy 113, 425-435 (2018)

22. A. Santamarina, D. Bernard, Y. Rugama, JEFF Report 22 (2009)

23. K. Shibata et al., J. Nucl. Sci. Technol. 48(1), 1-30 (2011)

24. M.B. Chadwick, Nuclear Data Sheets 112, 2887-2996 (2011)

25. G. Audi et al., Nucl. Phys. A729 3-129 (2003)

26. P. Moller, B. Pfeiffer, K.-L. Kratz, Phys. Rev. C. 67055802 (2003)

27. V. M. Piksaikin et al., , Prog. Nucl. Energy 41, 203-222 (2002)

28. A. Kochetkov et al., EPJ Web of Conferences 106, 06004 (2016)

29. A. Kochetkov et al., Proc. PHYSOR-2018, Cancun, Mexico

30. J. L. Lecouey et al., Ann. Nucl. Energy 83, 65-75 (2015)

31. J. Wagemans et al., EPJ Web of Conferences 170, 04027 (2018)

32. X-5 Monte Carlo Team, LA-UR-03-1987 (2003)

33. B. C. Kiedrowski, F. B. Brown, P. P. H. Wilson, Nucl. Sci. Eng. 168, 226-241 (2011)

34. B. C. Kiedrowski et al., LA-UR-10-06217 (2010)

35. E. Fridman, A. Kochetkov, A. Krása, Ann. Nucl. Energy 108, 239-252 (2017)

36. A. J. Koning et al., J. Korean Phys. Soc. 59, 1057-1062 (2011)

37. A. J. M. Plompen et al., to be published in EPJ

38. D. A. Brown et al., Nucl. Data Sheets 148, 1-142 (2018)

39. X. Doligez et al., IEEE Nucl. Sci. Symp. Conf. Rec, Proc. ANIMMA-2015 (2015) 